\title{
Low-frequency ultrasound and microbubbles combined with simvastatin promote the apoptosis of MCF-7 cells by affecting the LATS1/YAP/RHAMM pathway
}

\author{
$\mathrm{HAIGE} \mathrm{LI}^{1 *}, \mathrm{CHEN} \mathrm{CHEN}^{1 *}$ and DEHANG WANG ${ }^{2}$ \\ ${ }^{1}$ Department of Imaging, The Second Affiliated Hospital of Nanjing Medical University, Nanjing, Jiangsu 210011; \\ ${ }^{2}$ Department of Imaging, The First Affiliated Hospital of Nanjing Medical University, Nanjing, Jiangsu 210029, P.R. China
}

Received August 15, 2017; Accepted December 13, 2017

DOI: $10.3892 / \mathrm{mmr} .2018 .9273$

\begin{abstract}
Ultrasound scanning has widespread used in clinical practice and also has therapeutic applications. Simvastatin is a statins that is able to competitively inhibit the activity of 3-hydroxy-3-methylglutaryl-coenzyme A reductase. The aim of the present study was to investigate the roles and mechanisms of low-frequency ultrasound (LFU) and microbubbles combined with simvastatin on MCF-7 cell growth and apoptosis. Cell viability, apoptosis and cell cycle were evaluated using an MTT assay and flow cytometry, respectively. The expression of related proteins was measured by western blot assay. The results revealed that simvastatin and LFU with microbubbles reduces the viability of MCF-7 cells. The combination of LFU and microbubbles with simvastatin promoted the apoptosis of MCF-7 cells. Furthermore, it was confirmed that LFU and microbubbles combined with simvastatin affected the large tumor suppressor 1 (LATS1)/yes-associated protein (YAP)/receptor of the hyaluronan-mediated motility (RHAMM) pathway in MCF-7 cells. It was determined that LATS1 acts as a negative regulator in the LATS1/YAP/RHAMM pathway in MCF-7 cells. In conclusion, the results of the present study indicate that LFU and microbubbles combined with simvastatin promotes the apoptosis of MCF-7 cells via the LATS1/YAP/RHAMM pathway. The present study suggested a possible strategy for the treatment of breast cancer.
\end{abstract}

Correspondence to: Dr Dehang Wang, Department of Imaging, The First Affiliated Hospital of Nanjing Medical University, 300 Guangzhou Road, Nanjing, Jiangsu 210029, P.R. China

E-mail: dehangwang@163.com

*Contributed equally

Key words: low-frequency ultrasound, microbubbles, simvastatin, Michigan cancer foundation-7 cells, large tumor suppressor 1, yes-associated protein, receptor for the hyaluronan-mediated motility

\section{Introduction}

Breast cancer is the most common disease and the leading cause of cancer-associated mortality in women worldwide (1). At present, several strategies are employed for the treatment of early breast cancer, including surgical resection, chemotherapy, radiotherapy, endocrine drug therapy and targeted drug therapy (2). However, there is no effective way to treat advanced breast cancer (3). Relapse and metastasis of early breast cancer are frequent, and so existing treatments only improve the clinical symptoms, quality of life and overall survival rates of patients with breast cancer (4). The mechanisms of breast cancer require further innovation and exploration in order to develop novel and effective treatments.

The Hippo signaling pathway is a conserved kinase cassette, which controls the growth of tissues via activation of the yes-associated protein (YAP) and transcriptional co-activator with PDZ-binding motif (TAZ) effectors in humans (5-7). Transcriptional coactivators, including YAP and TAZ, are able to upregulate the expression of proliferative and anti-apoptotic genes $(8,9)$. Large tumor suppressor 1 (LATS1) is an upstream protein of YAP, which regulates YAP by excluding it from the nuclear compartment (10). LATS1 inactivation results in uncontrolled nuclear accumulation of YAP and may lead to the development of assorted cancers (11-13). It has been demonstrated that the interaction between mevalonate and the Hippo pathway regulates transcription of the receptor for hyaluronan-mediated motility (RHAMM) via YAP to modulate breast cancer cell motility (14). Extracellular regulated protein kinases (ERK), the protein kinase B (AKT) and mammalian target of rapamycin (mTOR) serve as the downstream proteins of the LATS1/YAP/RHAMM pathway (15-17). Based on these previous studies, it was hypothesized in the present study that the LATS1/YAP/RHAMM pathway serves a critical role in the development of breast cancer.

Statins, a class of 3-hydroxy-3-methylglutaryl-coenzyme A (HMG-CoA) reductase inhibitors, are able to competitively inhibit the activity of $\mathrm{HMG}-\mathrm{CoA}$ reductase. A previous study indicated that statins significantly decrease the incidence of lung, prostate, ovarian and colorectal cancers (18). 
Furthermore, studies have identified that statins may exert their anti-neoplastic impacts by affecting the c-Jun $\mathrm{N}$-terminal kinase/nuclear factor $\kappa$-light-chain-enhancer of activated B cells pathway (19), the phosphoinositide 3-kinase/AKT pathway (20) and the mitogen-activated protein kinase kinase/ERK pathway (21). It also has been demonstrated that the application of lipophilic statins, particularly simvastatin, reduces the risk of breast cancer (22). Studies have reported that simvastatin affects the ERK, AKT and mTOR pathways in several types of cancer cells $(23,24)$. Nevertheless, little is known about the exact mechanisms of simvastatin in the pathogenesis of breast cancer.

Though historically used for diagnostic purposes, ultrasound has recently become a therapeutic tool. The advantages of ultrasound therapy are that it is inexpensive, non-invasive and safe. Studies have identified that, compared with normal cells, cancer cells are more vulnerable to ultrasound $(25,26)$. Based on this, ultrasound has been used as a cancer treatment. A previous study confirmed that low-frequency ultrasound (LFU) facilitates the transport of macromolecules into the cell by increasing the permeability of the cell membrane (27). Through the cavitation effect, LFU may unite with microbubbles to enhance its anticancer properties (28). Additionally, ultrasound united with microbubbles has been demonstrated to exert anticancer effects in multiple cancer cells, including prostate, ovarian and hepatocellular carcinomas (29-31). However, the roles and mechanisms of LFU with microbubbles on breast cancer remain to be elucidated.

The aim of the present study was to investigate the functions and mechanisms of LFU and microbubbles combined with simvastatin on the growth, cell cycle and apoptosis of MCF-7 cells. The association between LFU and microbubbles combined with simvastatin and the LATS1/YAP/RHAMM pathway in MCF-7 cells was also investigated.

\section{Materials and methods}

Reagents. All the products used in cell culture were obtained from Gibco (Thermo Fisher Scientific, Inc., Waltham, MA, USA). Antibodies, LATS1 small interfering (si)RNAs and LATS1 negative siRNAs were purchased from Abcam (Cambridge, UK). Simvastatin was purchased from Pfizer, Inc. (New York, NY, USA) and the solvent of simvastatin was dimethyl sulfoxide (DMSO; Xilong Scientific Co., Ltd., Shenzhen, China). Microbubbles used in the present study were obtained from Definity (Lantheus Medical Imaging, Inc., North Billerica, MA, USA). The shell material, gas, mean size, concentration and microbubble half-life were phospholipid, $\mathrm{C}_{3} \mathrm{~F}_{8}, 1.1-3.3 \mu \mathrm{m}, 1.2 \times 10^{10}$ bubbles $/ \mathrm{ml}$ and 2-10 min, respectively. Based on previous studies, the concentration of microbubbles used in the present study was chosen as $20 \%$; the ratio of microbubble solution: Cell suspension was $2: 8(32,33)$.

Cell culture and grouping. The human breast cancer cell line MCF-7 was purchased from GefanBio (Shanghai, China). Cells were routinely maintained in Dulbecco's modified Eagle's medium (DMEM; Gibco; Thermo Fisher Scientific, Inc.) supplemented with $10 \%$ fetal bovine serum (FBS;
Gibco; Thermo Fisher Scientific, Inc.). A total of four treatment groups were prepared for the present study as follows: Control group, MCF-7 cells without any treatment or the addition of any solvent; SIM group, MCF-7 cells treated with $12 \mu \mathrm{M}$ simvastatin for $18 \mathrm{~h}$ at $37^{\circ} \mathrm{C}$; UM group, MCF-7 cells treated with $0.6 \mathrm{~W} / \mathrm{cm}^{2}$ ultrasound and $20 \%$ microbubbles; and UM+SIM group, MCF-7 cells treated with $0.6 \mathrm{~W} / \mathrm{cm}^{2}$ ultrasound and $20 \%$ microbubbles combined with $12 \mu \mathrm{M}$ simvastatin. For transfection experiments, the UM+SIM group was used and two additional groups were prepared as follows: UM+SIM+LATS1 siRNAs group, MCF-7 cells treated with $0.6 \mathrm{~W} / \mathrm{cm}^{2}$ ultrasound and $20 \%$ microbubbles, $12 \mu \mathrm{M}$ simvastatin and $25 \mathrm{nM}$ LATS1 small interfering (si)RNA (GCAATCAGTTAACCGCAA; Invitrogen; Thermo Fisher Scientific, Inc.); and UM+SIM+LATS1 negative siRNAs group, MCF-7 cells treated with $0.6 \mathrm{~W} / \mathrm{cm}^{2}$ ultrasound and $20 \%$ microbubbles, $12 \mu \mathrm{M}$ simvastatin and $25 \mathrm{nM}$ LATS1 negative siRNA (GCACAGTTAACCGCATAAA; Invitrogen; Thermo Fisher Scientific, Inc.). The transfection regent Lipofectamine ${ }^{\circledR} 3000$ was purchased from Invitrogen (Thermo Fisher Scientific, Inc.). Following transfection, the cells were maintained at $37^{\circ} \mathrm{C}$ for $24 \mathrm{~h}$ and then were subject to the subsequent experiments.

Cell viability analysis. Cell viability was measured using an MTT assay. Briefly, MCF-7 cells in the logarithmic phase were seeded $\left(\sim 5 \times 10^{4}\right.$ cells $\left./ \mathrm{ml}\right)$ in 96 -well plates and maintained for $12 \mathrm{~h}$ in an incubator at $37^{\circ} \mathrm{C}$ in an atmosphere containing $5 \% \mathrm{CO}_{2}$. Cells were treated with simvastatin at $1,3,6,12,25$ and $50 \mu \mathrm{M}$ and ultrasound (Antares ultrasound scanner, Siemens Medical Solutions USA Inc., Malvern, PA, USA) united microbubbles (20\%; Lantheus Medical Imaging, Inc.) at $0.1,0.3,0.6,1.0$ and $1.5 \mathrm{~W} / \mathrm{cm}^{2}$. Cells were centrifuged at $224 \mathrm{x} \mathrm{g}$ for $1 \mathrm{~min}$ at room temperature and the supernatant was removed. Cells were then treated with $100 \mu \mathrm{l}$ DMSO under low-speed oscillation $(400 \mathrm{rpm} / \mathrm{min})$ for $10 \mathrm{~min}$ at room temperature. Absorbance was detected at $490 \mathrm{~nm}$ using a microplate reader (Bio-Rad Laboratories, Inc., Hercules, CA, USA).

Flow cytometry. Cell apoptosis was assessed by flow cytometry (FCM). MCF-7 cells were treated as described above. Following washing with PBS (twice, 2-3 min each time), MCF-7 cells were trypsinized using $0.25 \%$ trypsin (Beyotime Institute of Biotechnology, Haimen, China) and centrifuged at $224 \mathrm{x} \mathrm{g}$ for $1 \mathrm{~min}$ at $4^{\circ} \mathrm{C}$. The apoptosis detection kit with Annexin $\mathrm{V}$ FITC and PI was purchased from Invitrogen (Thermo Fisher Scientific, Inc.). The supernatant was removed and cells were suspended in the Annexin-binding buffer provided by the kit at a density of $1 \times 10^{6}$ cells $/ \mathrm{ml}$. The cells were then incubated with Annexin V-fluorescein isothiocyanate and propidium iodide (PI; provided by the kit) at room temperature for $15 \mathrm{~min}$ in the dark. Cell apoptosis was assessed using a FACSCalibur flow cytometer with CellQuest analysis software version 3.3 (BD Biosciences, San Diego, CA, USA).

Cell cycle analysis. MCF-7 cells in the logarithmic phase were seeded $\left(\sim 6 \times 10^{7}\right.$ cells/well) in 6 -well plates and maintained for $24 \mathrm{~h}$ in an incubator at $37^{\circ} \mathrm{C}$ in an atmosphere containing $5 \% \mathrm{CO}_{2}$, treated as described above and maintained in an 

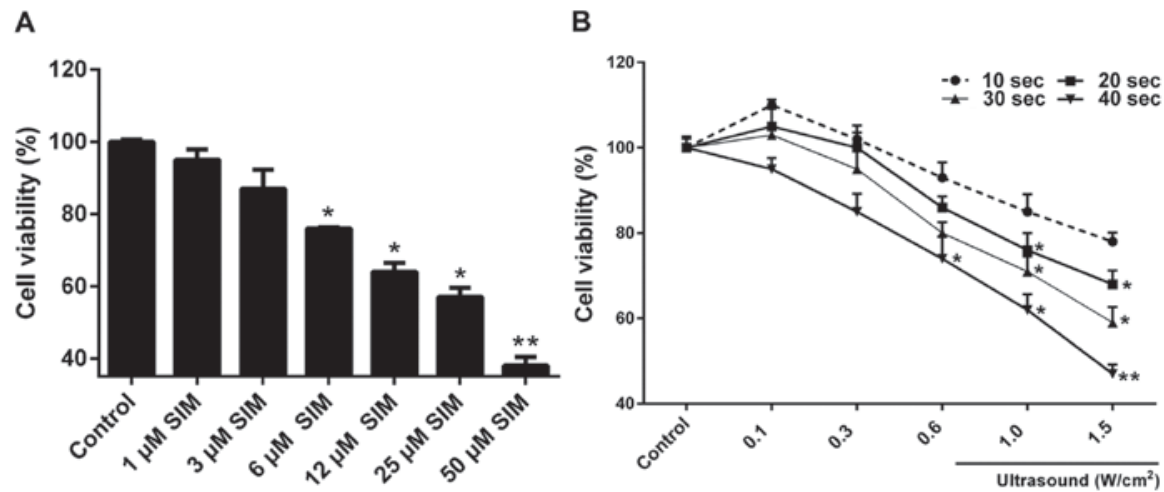

Figure 1. SIM and LFU with microbubbles suppresses the viability of MCF-7 cells. MTT assays was performed to assess the viability of MCF-7 cells treated with (A) 1,3, 6, 12, 25 and $50 \mu \mathrm{M}$ SIM or (B) LFU and microbubbles (20\%) at 0.1, 0.3, 0.6, 1.0 and $1.5 \mathrm{~W} / \mathrm{cm}^{2}$ for $10,20,30$ and 40 sec. ${ }^{*} \mathrm{P}<0.05$ and ${ }^{* *} \mathrm{P}<0.01$ vs. control. LFU, low-frequency ultrasound; SIM, simvastatin.

incubator at $37^{\circ} \mathrm{C}$ in an atmosphere containing $5 \% \mathrm{CO}_{2}$ for $48 \mathrm{~h}$. The medium was removed and cells were washed three times with PBS (3 min each time). Following digestion with $0.25 \%$ trypsin, the cell suspension was collected and centrifuged for $5 \mathrm{~min}$ at $224 \mathrm{x} \mathrm{g}$ at $4^{\circ} \mathrm{C}$ and the supernatant was discarded. Following washing with PBS 3 times (3 min each time), the cells were re-suspended, centrifuged for $5 \mathrm{~min}$ at $224 \mathrm{x} \mathrm{g}$ at $4^{\circ} \mathrm{C}$ and the supernatant was discarded once more. Pre-cooled $70 \%$ ethanol $(1 \mathrm{ml})$ was added to cells, which were subsequently stored in at $4^{\circ} \mathrm{C}$ overnight and centrifuged for $5 \mathrm{~min}$ at $224 \mathrm{x} \mathrm{g}$. The ethanol was removed and cells were washed three times with PBS (3 min each time). A total $500 \mu$ PBS containing PI $(50 \mu \mathrm{g} / \mathrm{ml})$, RNase A $(100 \mu \mathrm{g} / \mathrm{ml})$ and Triton X-100 $(0.2 \%)$ was added to the cells for $30 \mathrm{~min}$ in the dark. FCM was performed as above and FlowJo software version 7.2 (FlowJo LLC, Ashland, OR, USA) was used for cell cycle analysis according to the manufacturer's protocol.

Western blot analysis. Cultured cells were lysed on ice in radioimmunoprecipitation assay lysis buffer $\left(1 \mathrm{mM} \mathrm{MgCl}_{2}\right.$, $1 \%$ Triton $\mathrm{X}-100,10 \mathrm{mM}$ Tris- $\mathrm{HCl}$ and $0.1 \%$ SDS, $\mathrm{pH}$ 7.4) and fragments using an ultrasonic cell disruptor (Branson Ultrasonics Corp., Danbury, CT, USA). The fragments were removed and the supernatant liquor was collected by centrifugation at $224 \mathrm{xg}$ for $5 \mathrm{~min}$ at $4^{\circ} \mathrm{C}$. The concentration of proteins was established using a Bradford protein assay kit (Bio-Rad Laboratories, Inc.) following the manufacturer's protocol. Equal quantities of proteins $(50 \mu \mathrm{g})$ were separated by $5 \%$ SDS-PAGE and transferred onto nitrocellulose membranes (EMD Millipore, Billerica, MA, USA). Following washing with PBS for 3 times (5 min each time), nonspecific binding blocked with 5\% low fat dried milk at room temperature for $2 \mathrm{~h}$. Membranes were incubated overnight with the following primary antibodies: Anti-phosphorylated (p-)ERK (ab65142; 1:800), anti-p-AKT (ab18206; 1:1,000), anti-p-mTOR (ab84400; $1: 1,000)$, anti- $\beta$-actin (ab8227; 1:2,000), anti-LATS1 (ab70561; 1:8,000), anti-YAP (ab39361; 1:1,000), anti-p-YAP (ab153077; 1:500), anti-RHAMM (ab143134; 1:1,000), anti-Kruppel-like factor 5 (KLF5; ab137676; 1:2,000) at $4^{\circ} \mathrm{C}$. Then, horseradish peroxidase-conjugated secondary antibodies (bs-0293M; 1:1,000; BIOSS, Beijing, China) were added at room temperature for $1 \mathrm{~h}$. The results were quantified using EMD Millipore $^{\mathrm{TM}}$ Immobilon $^{\mathrm{TM}}$ Western Chemiluminescent
HRP Substrate (EMD Millipore). The data were analyzed by ImageJ version 1.48 (National Institutes of Health, Bethesda, MD, USA).

Statistical analysis. Data are presented as the mean \pm standard deviation. The results were analyzed by one-way analysis of variance followed by Turkey's test. $\mathrm{P}<0.05$ was considered to indicate a statistically significant difference.

\section{Results}

Simvastatin and LFU combined with microbubbles reduces the viability of MCF-7 cells. MTT results revealed that simvastatin reduced the viability of MCF-7 cells in a dose-dependent manner (Fig. 1A). In addition, it was indicated that simvastatin decreased the viability of MCF-7 cells treated with different intensities and durations of LFU and microbubbles (Fig. 1B). Thus, it was confirmed that simvastatin and LFU combined with microbubbles is able to suppress the viability and growth of MCF-7 cells.

LFU and microbubbles combined with simvastatin arrest MCF-7 cells in G1 phase and induced the apoptosis of MCF-7 cells. Cell cycle arrest and apoptosis of MCF-7 cells treated with LFU and microbubbles combined with simvastatin was evaluated. The results demonstrated that the percentage of cells in G1 phase in the UM+SIM group $(82.47 \%)$ was higher compared with other groups (CON, 58.32\%; SIM, 78.34\%; UM, 65.90\%; Fig. 2). This indicates that LFU and microbubbles combined with simvastatin induced cell cycle arrest at G1 phase. FCM data (Fig. 3) revealed that the percentage of apoptotic cells in the UM+SIM group (35.63\%) was significantly higher compared with the CON (2.30\%), SIM (23.64\%) and UM groups (9.61\%). This suggests that LFU and microbubbles combined with simvastatin induces apoptosis in MCF-7 cells.

LFU and microbubbles combined with simvastatin affect the LATS1/YAP/RHAMM pathway. The expression of LATS1, YAP and RHAMM, together with their downstream proteins, was investigated in MCF-7 cells. In the UM+SIM group, the levels of YAP and RHAMM were significantly lower compared with the control (Fig. 4A). However, an increase in LATS1 and 

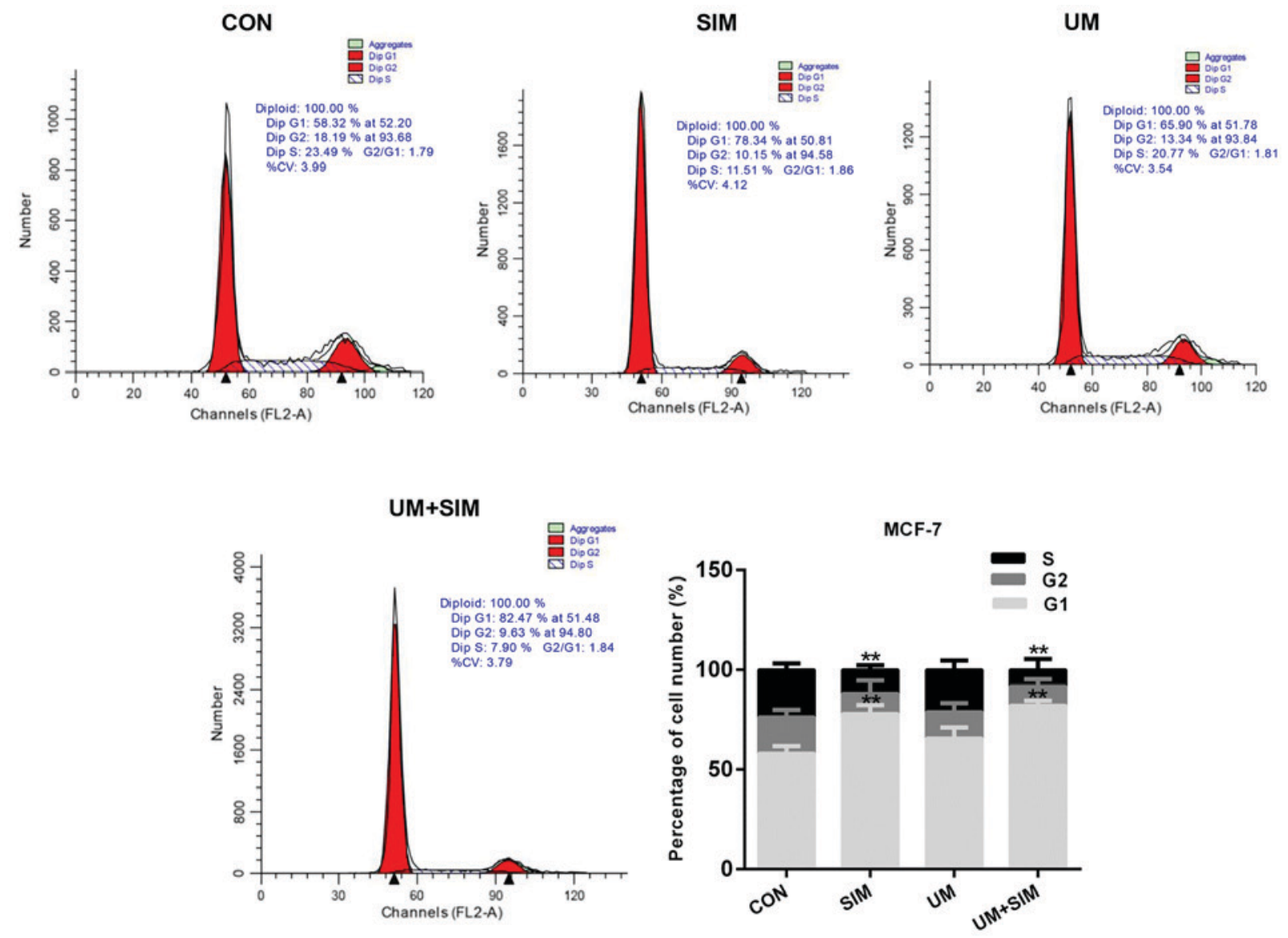

Figure 2. LFU and microbubbles combined with simvastatin induces cell cycle arrest in G1 phase. Flow cytometry was performed to analyze the cell cycle distribution of MCF-7 cells in the CON, SIM, UM and UM+SIM groups. ${ }^{* *} \mathrm{P}<0.01$ vs. CON. LFU, low-frequency ultrasound; CON, control; SIM, MCF-7 cells treated with $12 \mu \mathrm{M}$ simvastatin; UM, cells treated with $0.6 \mathrm{~W} / \mathrm{cm}^{2}$ ultrasound and $20 \%$ microbubbles; UM+SIM, MCF-7 cells treated with $0.6 \mathrm{~W} / \mathrm{cm}^{2}$ ultrasound and $20 \%$ microbubbles combined with $12 \mu \mathrm{M}$ simvastatin.

p-YAP expression was observed in MCF-7 cells treated with LFU and microbubbles combined with simvastatin compared with control cells (Fig. 4A). These results indicate that LFU and microbubbles combined with simvastatin downregulates the expression of YAP and RHAMM, upregulates LATS1 expression and promotes the phosphorylation of YAP in MCF-7 cells. KLF5, a downstream protein of YAP, had the lowest expression in the UM+SIM group (Fig. 4A). In addition, the expression of downstream proteins associated with LATS1/YAP/RHAMM pathway in MCF-7 cells is presented in Fig. 4. Compared with other groups, the expression of phosphorylated ERK, AKT and mTOR was lowest in MCF-7 cells treated with LFU and microbubbles combined with simvastatin (Fig. 4B). However, no significant difference was observed ERK, AKT and mTOR expression between groups. It was demonstrated that LFU and microbubbles combined with simvastatin enhanced LATS1 expression and reduced YAP and RHAMM expression in MCF-7 cells. In addition, LFU and microbubbles combined with simvastatin further downregulated the expression of downstream proteins associated with LATS1/YAP/RHAMM, involving ERK, AKT and mTOR. These data suggest that LFU and microbubbles combined with simvastatin affects the LATS1/YAP/RHAMM pathway in MCF-7 cells. pathway in MCF-7 cells. MTT results (Fig. 5) revealed that the viability of MCF-7 cells in the UM+SIM+LATS1 siRNA group was increased compared with other groups. Furthermore, it was identified that the viability of MCF-7 cells in the UM+SIM and $\mathrm{UM}+\mathrm{SIM}+\mathrm{LATS} 1$ negative siRNA groups was very similar, which suggests that LATS1 suppresses the growth of MCF-7 cells. It was also identified that, compared with the other groups, the apoptosis of MCF-7 cells was suppressed in the UM+SIM+LATS1 siRNA group (Fig. 5B and C). These data indicate that LATS1 promotes the apoptosis of MCF-7 cells. In order to verify this, the expression of proteins associated with the LATS1/YAP/RHAMM pathway was further assessed. The results demonstrated that, in the UM+SIM+LATS1 siRNAs group, the expression of LATS1 and p-YAP in was lower compared with other groups. However, YAP, RHAMM and KLF5 expression in MCF-7 cells was upregulated in the UM+SIM+LATS1 siRNA group (Fig. 6A). The expression of p-ERK, AKT and mTOR in the UM+SIM+LATS1 siRNA group was higher compared with other groups (Fig. 6B). As the upregulation of LATS1 expression was accompanied by a decrease in YAP and RHAMM expression, it was confirmed that LATS1 negatively regulates the expression of YAP and RHAMM in MCF-7 cells. These data suggest that LATS1 acts as a negative regulator in the LATS1/YAP/RHAMM pathway in MCF-7 cells. Furthermore, a hypothetical model of the pathway and indirect role of LATS1 in MCF-7 cells was established (Fig. 7). 

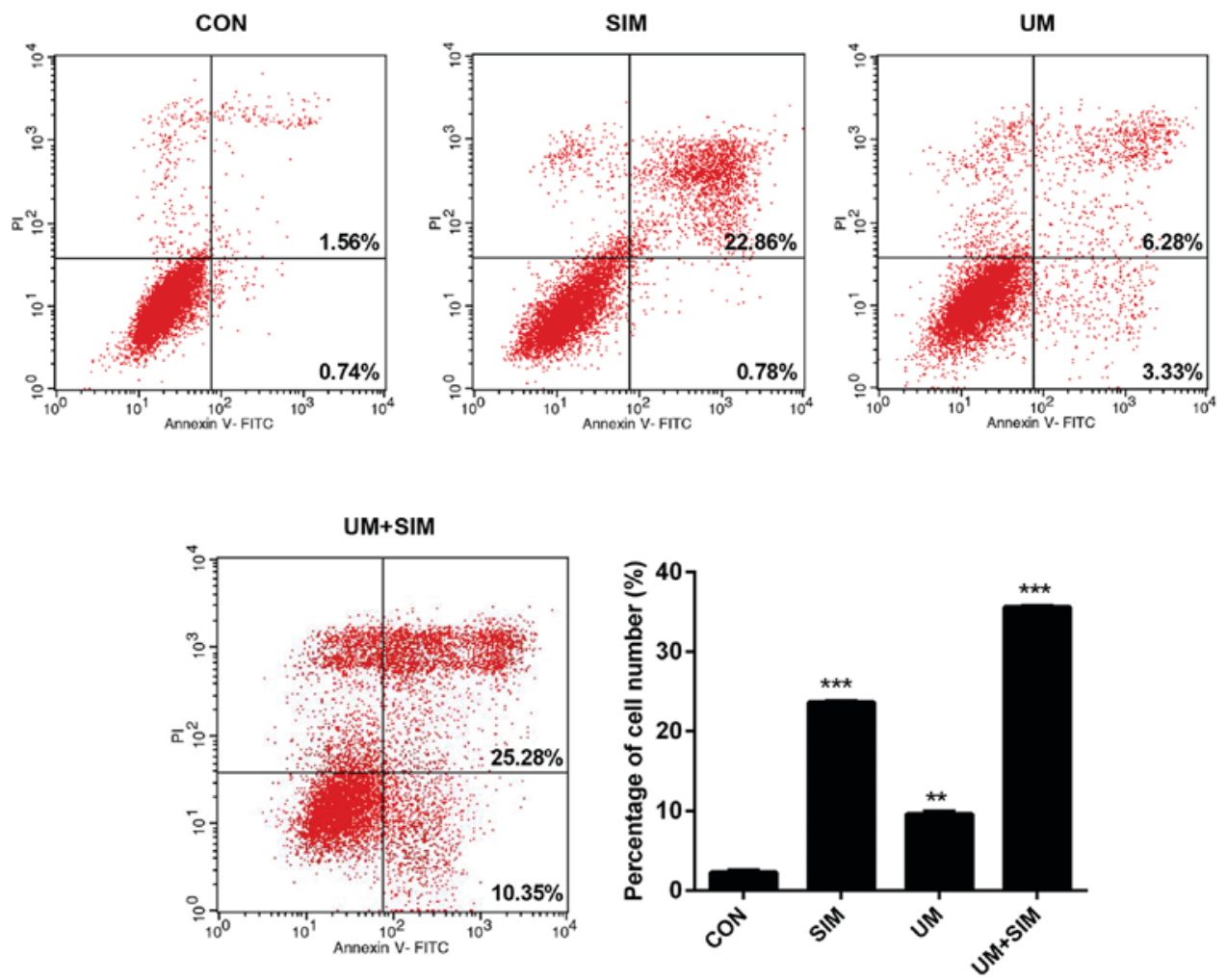

Figure 3. LFU and microbubbles combined with simvastatin induces apoptosis in MCF-7 cells. Flow cytometry was performed to assess the apoptosis of MCF-7 cells in the CON, SIM, UM and UM+SIM groups. ${ }^{* *} \mathrm{P}<0.01$ and ${ }^{* * * *} \mathrm{P}<0.001$ vs. CON. LFU, low-frequency ultrasound; CON, control; SIM, MCF-7 cells treated with $12 \mu \mathrm{M}$ simvastatin; UM, cells treated with $0.6 \mathrm{~W} / \mathrm{cm}^{2}$ ultrasound and $20 \%$ microbubbles; UM+SIM, MCF-7 cells treated with $0.6 \mathrm{~W} / \mathrm{cm}^{2}$ ultrasound and $20 \%$ microbubbles combined with $12 \mu \mathrm{M}$ simvastatin; FITC, fluorescein isothiocyanate.

A
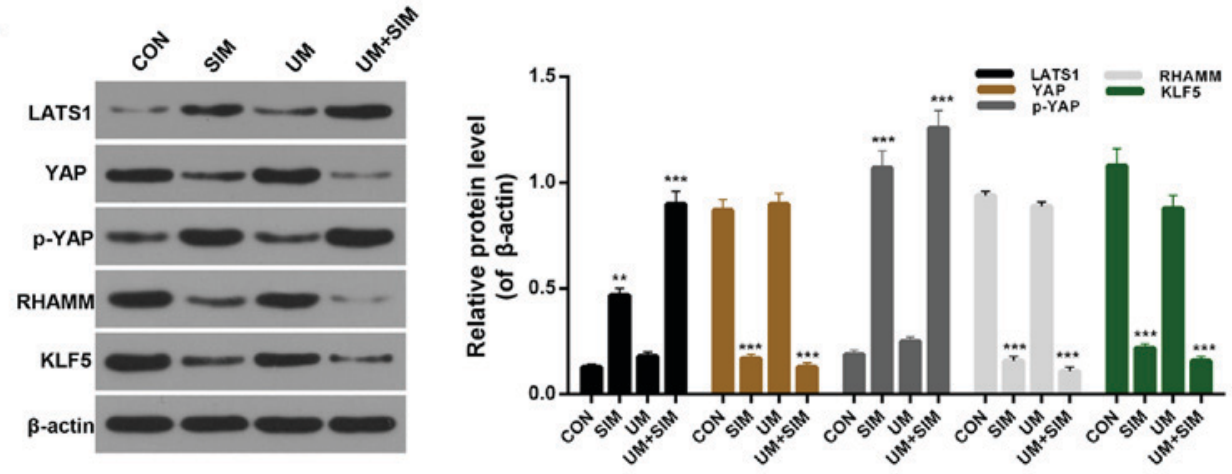

B
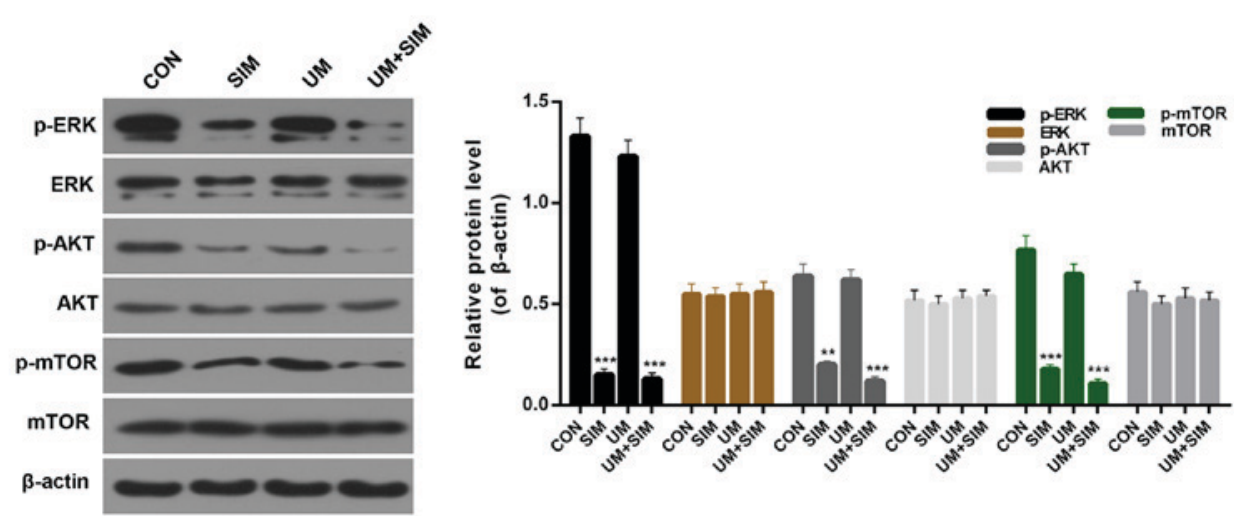

Figure 4.LFU and microbubbles combined with simvastatin affects the LATS1/YAP/RHAMM pathway in MCF-7 cells. (A) Western blot analysis was performed to assess the expression of LATS1, YAP, p-YAP, RHAMM and KLF5 and (B) p-ERK, ERK, p-AKT, AKT, p-mTOR and mTOR in MCF-7 cells in the CON, SIM, UM and UM+SIM groups. ${ }^{* *} \mathrm{P}<0.01$ and ${ }^{* * * *} \mathrm{P}<0.001$ vs. control. LFU, low-frequency ultrasound; LATS1, large tumor suppressor 1; YAP, yes-associated protein; RHAMM, receptor for the hyaluronan-mediated motility; p, phosphorylated; KLF5, Kruppel-like factor 5; ERK, extracellular regulated protein kinases; AKT, protein kinase B; mTOR, mammalian target of rapamycin; CON, control; SIM, MCF-7 cells treated with $12 \mu \mathrm{M}$ simvastatin; UM, cells treated with $0.6 \mathrm{~W} / \mathrm{cm}^{2}$ ultrasound and 20\% microbubbles; UM+SIM, MCF-7 cells treated with $0.6 \mathrm{~W} / \mathrm{cm}^{2}$ ultrasound and $20 \%$ microbubbles combined with $12 \mu \mathrm{M}$ simvastatin. 
A

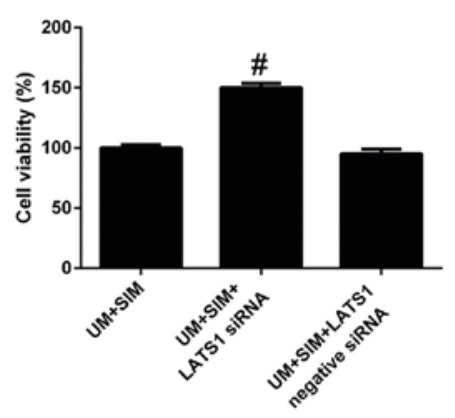

B

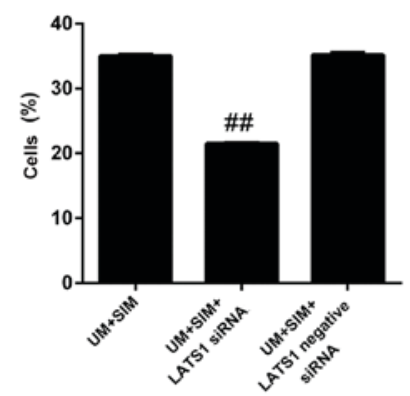

C
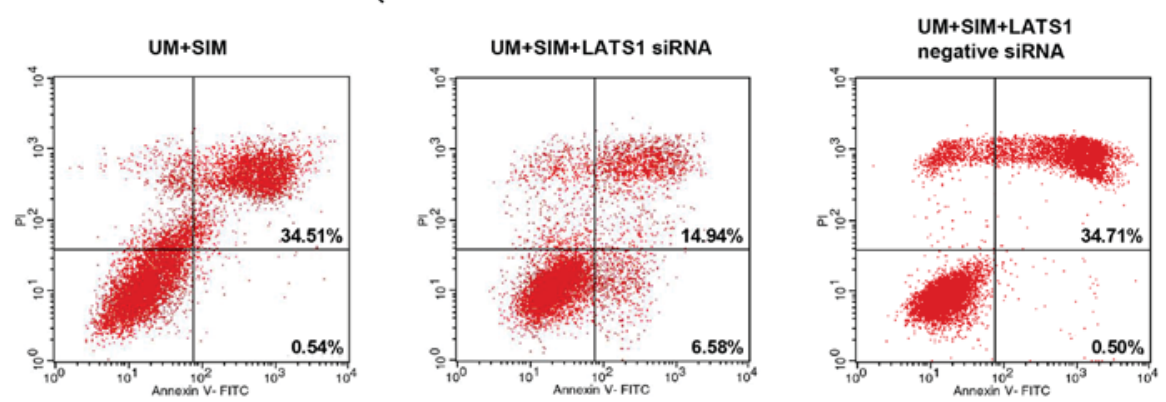

Figure 5. LATS1 affects the viability and apoptosis of MCF-7 cells. (A) An MTT assay and (B and C) flow cytometry was performed to assess the viability and apoptosis, respectively, of MCF-7 cells in the UM+SIM, UM+SIM+LATS1 siRNA and UM+SIM+LATS1 negative siRNA groups. ${ }^{\# P<0.05}$ and ${ }^{\# \#} \mathrm{P}<0.01$ vs. UM+SIM+LATS1 negative siRNAs. LATS1, large tumor suppressor 1; LFU, low-frequency ultrasound; siRNA, small interfering RNA; UM+SIM, MCF-7 cells treated with $0.6 \mathrm{~W} / \mathrm{cm} 2$ ultrasound and $20 \%$ microbubbles combined with $12 \mu \mathrm{M}$ simvastatin; UM+SIM+LATS1 siRNAs, MCF-7 cells treated with $0.6 \mathrm{~W} / \mathrm{cm}^{2}$ ultrasound and $20 \%$ microbubbles, $12 \mu \mathrm{M}$ simvastatin and $25 \mathrm{nmol} / \mathrm{ml}$ LATS1 siRNA; UM+SIM+LATS1 negative siRNAs, MCF-7 cells treated with $0.6 \mathrm{~W} / \mathrm{cm}^{2}$ ultrasound and $20 \%$ microbubbles, $12 \mu \mathrm{M}$ simvastatin and $25 \mathrm{nmol} / \mathrm{ml}$ LATS1 negative siRNA; FITC, fluorescein isothiocyanate.
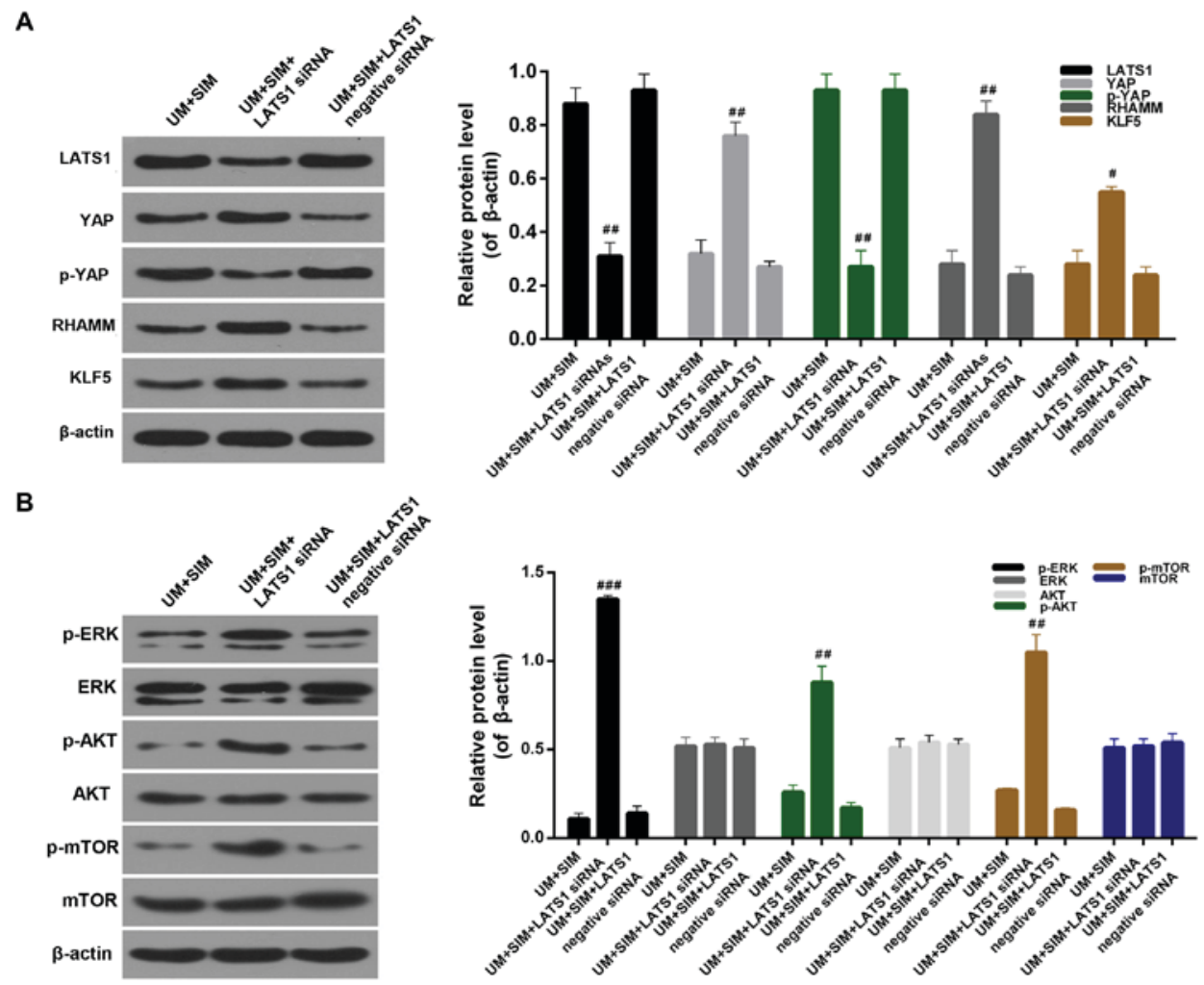

Figure 6. LATS1 serves as a negative regulator in the LATS1/YAP/RHAMM pathway in MCF-7 cells. Western blot assays was performed to measure the expression of (A) LATS1, YAP, p-YAP, RHAMM and KLF5 and (B) p-ERK, ERK, p-AKT, AKT, p-mTOR and mTOR in MCF-7 cells in the UM+SIM, $\mathrm{UM}+\mathrm{SIM}+\mathrm{LATS} 1$ siRNA and UM+SIM+LATS1 negative siRNA groups. ${ }^{*} \mathrm{P}<0.05,{ }^{\# \#} \mathrm{P}<0.01$ and ${ }^{\# \# \#} \mathrm{P}<0.001 \mathrm{vs}$. UM+SIM+LATS1 negative siRNAs. LATS1, large tumor suppressor 1; YAP, yes-associated protein; RHAMM, receptor for the hyaluronan-mediated motility; siRNA, small interfering RNA; p, phosphorylated; KLF5, Kruppel-like factor 5; ERK, extracellular regulated protein kinases; AKT, protein kinase B; mTOR, mammalian target of rapamycin; $\mathrm{UM}+\mathrm{SIM}, \mathrm{MCF}-7$ cells treated with $0.6 \mathrm{~W} / \mathrm{cm}^{2}$ ultrasound and $20 \%$ microbubbles combined with $12 \mu \mathrm{M}$ simvastatin; UM+SIM+LATS1 siRNAs, MCF-7 cells treated with $0.6 \mathrm{~W} / \mathrm{cm}^{2}$ ultrasound and $20 \%$ microbubbles, $12 \mu \mathrm{M}$ simvastatin and $25 \mathrm{nmol} / \mathrm{ml}$ LATS1 siRNA; UM+SIM+LATS1 negative siRNAs, MCF-7 cells treated with $0.6 \mathrm{~W} / \mathrm{cm}^{2}$ ultrasound and $20 \%$ microbubbles, $12 \mu \mathrm{M}$ simvastatin and $25 \mathrm{nmol} / \mathrm{ml}$ LATS1 negative siRNA. 

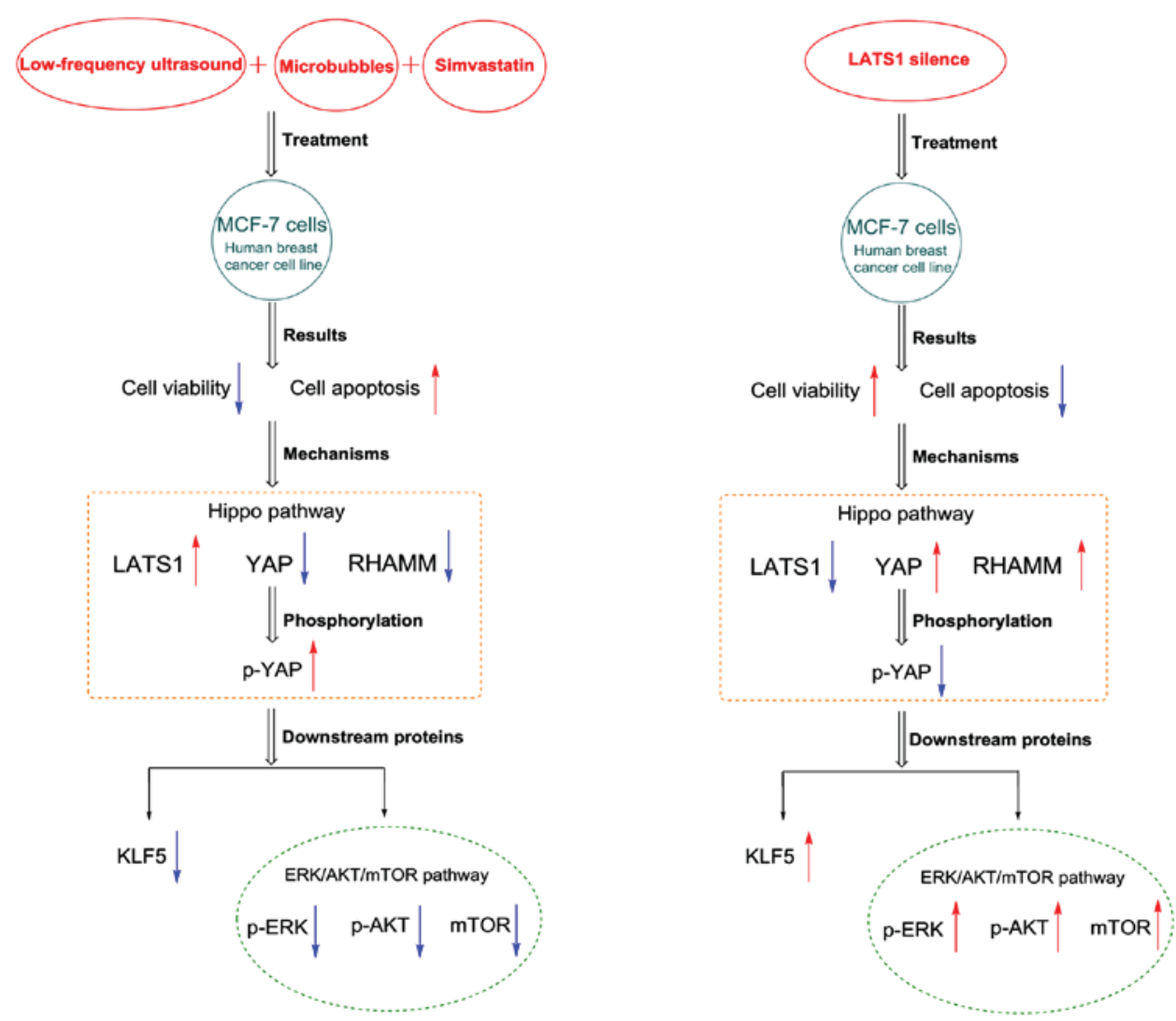

Figure 7. Schematic overview of LATS1/YAP/RHAMM pathway and the regulatory effects of LATS1 in the LATS1/YAP/RHAMM pathway. LATS1, large tumor suppressor 1; YAP, yes-associated protein; RHAMM, receptor for the hyaluronan-mediated motility; p, phosphorylated; KLF5, Kruppel-like factor 5; ERK, extracellular regulated protein kinases; AKT, protein kinase B; mTOR, mammalian target of rapamycin.

\section{Discussion}

The biological influence of LFU and microbubbles on cancer cells is dependent on the microbubble density, ultrasound intensity and irradiation time $(34,35)$. Generally, a pilot experiment should be prepared to debug the optimized ultrasound parameters, so as to induce cell apoptosis to the maximum extent $(36,37)$. Studies have identified that microbubbles enhance ultrasound exposure by decreasing the cavitation threshold (27), which induces cancer cell apoptosis $(38,39)$. In the present study, it was demonstrated that microbubbles used together with low-frequency, low-intensity and short-exposure ultrasound reduced the viability of MCF-7 cells.

Simvastatin is a member of the statins family, inhibitors of the mevalonate signaling pathway, which regulates the synthesis of cholesterol (40). Previous studies have reported that statins may be effective for the prevention and treatment of cancer due to their lipid-lowering effects $(41,42)$. In addition, a number of statins have been identified that inhibit the proliferation of human breast cancer cells in vitro (43). Thus, the viability of MCF-7 cells treated with simvastatin was assessed in the present study. The results revealed that simvastatin reduces the viability of MCF-7 cells in a dose-dependent manner. It has previously been demonstrated that simvastatin inhibits cell growth and induces apoptosis and G0/G1 cell cycle arrest in hepatic cancer cells (44). Nevertheless, at present, the effect of ultrasound in conjunction with microbubbles on cancer cells has yet to be reported. In the present study, the cell cycle distribution of MCF-7 cells treated with simvastatin and LFU united microbubbles was assessed. The results indicated that LFU and microbubbles combined with simvastatin induced MCF-7 cell cycle arrest in G1 phase. Previous studies have reported that simvastatin induces apoptosis in human breast and cancer cells $(45,46)$. The present research demonstrated that simvastatin induces apoptosis in MCF-7 cells and that the combination of ultrasound, microbubbles and simvastatin significantly promoted this effect. These results indicate that treatment with LFU and microbubbles combined with simvastatin has a greater anticancer effect compared with either treatment alone.

A number of protein kinases and signaling molecules are associated with the Hippo signaling pathway, including LATS1 (47), YAP (48) and RHAMM (14). KLF5 is a downstream protein of YAP, and is regulated by YAP (49). ERK, AKT and mTOR serve as downstream proteins of the LATS1/YAP/RHAMM pathway $(50,51)$. Previous studies have demonstrated that the Hippo signaling pathway in breast cancer cells may be influenced by Taxol, geranylgeranylation signals and mevalonate $(14,52,53)$. Additionally, it has been reported that simvastatin affects the ERK, AKT and mTOR pathways in several types of cancer cell $(23,24)$. Thus, the expression of proteins associated with the LATS1/YAP/RHAMM pathway in MCF-7 cells was assessed in the present study. The results revealed that, following treating with LFU and microbubbles combined with simvastatin, the expression of YAP, RHAMM, KLF5, p-ERK, p-AKT and p-mTOR was reduced, whereas LATS1 and p-YAP expression was increased in MCF-7 cells. These results indicate that LFU and microbubbles combined 
with simvastatin may affect the LATS1/YAP/RHAMM pathway.

In the present study it was conjectured whether LATS1 serves as a negative regulator in the LATS1/YAP/RHAMM pathway. In order to further investigate the regulatory mechanisms of the LATS1/YAP/RHAMM pathway, the cell viability and apoptosis of MCF-7 cells treated with LATS1 siRNAs and LATS1 negative siRNAs together with LFU and microbubbles combined with simvastatin was explored. The viability of MCF-7 cells was enhanced following LATS1 knockdown. It was also demonstrated that the apoptosis of MCF-7 cells treated with LATS1 siRNAs was distinctly reduced. These data suggest that LATS1 suppresses cell viability and induces apoptosis in MCF-7 cells. The expression of proteins associated with the LATS1/YAP/RHAMM pathway in MCF-7 cells treated with LATS1 siRNA and LATS1 negative siRNAs together with LFU and microbubbles combined with simvastatin was also evaluated. The results indicated that the expression of YAP, RHAMM, KLF5, p-ERK, p-AKT and p-mTOR in was downregulated following LATS1 knockdown, whereas, LATS1 and p-YAP expression was enhanced. Increases in LATS1 expression was always accompanied by a decrease in YAP and RHAMM expression, confirming that LATS1 negatively regulates the expression of YAP and RHAMM in MCF-7 cells. On the basis of these results, it may be hypothesized that LATS1 functions as a negative regulator of the LATS1/YAP/RHAMM pathway in MCF-7 cells. In addition, treatment with LFU and microbubbles combined with simvastatin induced an upregulation inLATS1 expression in MCF-7 cells and high levels of LATS1 further suppressed cell viability and promoted the apoptosis of MCF-7 cells.

Taken together, these results indicate that combined treatment with LFU and microbubbles and simvastatin promotes the apoptosis and decreases the viability of MCF-7 cells via the LATS1/YAP/RHAMM pathway. It was also demonstrated that LATS1 serves as a negative regulator in the LATS1/YAP/RHAMM pathway in MCF-7 cells. However, further studies are required to fully elucidate the roles and pivotal mechanisms of LFU and microbubbles combined with simvastatin in the progression and pathogenesis of breast cancer in order to develop effective therapeutic treatments.

In conclusion, to the best of the authors' knowledge, the present study is the first to demonstrate that LFU and microbubbles combined with simvastatin promotes the apoptosis of MCF-7 cells by affecting the LATS1/YAP/RHAMM pathway. Therefore, the findings of the present study may provide a novel insight into the pathogenesis of breast cancer and potential therapeutic methods.

\section{Acknowledgements}

Not applicable.

\section{Funding}

The present study was supported by the General Subject of Medical Science Development in Nanjing: Research on imaging mode of digital mammography and magnetic resonance in sub-clinical breast cancer (grant no. YKK14181).

\section{Availability of data and materials}

All data generated and/or analyzed during this study are included in this published article.

\section{Authors' contributions}

HL and DW designed the study. HL and CC performed the experiments. HL performed data analysis. HL wrote the manuscript. HL, CC and DW contributed to the manuscript revisions.

\section{Ethics approval and consent to participate}

Not applicable.

\section{Consent for publication}

Not applicable.

\section{Competing interests}

The authors declare that they have no competing interests.

\section{References}

1. Ferlay J, Parkin DM and Steliarova-Foucher E: Estimates of cancer incidence and mortality in Europe in 2008. Eur J Cancer 46: 765-781, 2010.

2. Goldhirsch A, Winer EP, Coates AS, Gelber RD, Piccart-Gebhart M, Thurlimann B and Senn HJ; Panel members: Personalizing the treatment of women with early breast cancer: Highlights of the St gallen international expert consensus on the primary therapy of early breast cancer 2013. Ann Oncol 24: 2206-2223, 2013

3. DeSantis C, Ma J, Bryan L and Jemal A: Breast cancer statistics, 2013. CA Cancer J Clin 64: 52-62, 2014

4. Miller KD, Siegel RL, Lin CC, Mariotto AB, Kramer JL, Rowland JH, Stein KD, Alteri R and Jemal A: Cancer treatment and survivorship statistics, 2016. CA Cancer J Clin 66: 271-289, 2016.

5. Enderle L and McNeill H: Hippo gains weight: Added insights and complexity to pathway control. Sci Signal 6: re7, 2013.

6. Halder G and Johnson RL: Hippo signaling: Growth control and beyond. Development 138: 9-22, 2011.

7. Staley BK and Irvine KD: Hippo signaling in Drosophila: Recent advances and insights. Dev Dyn 241: 3-15, 2012.

8. Svobodová J, Šmerdová L, Procházková J, Topinka J, Líbalová H, Machala M and Vondráček J: Induction of apoptosis in undifferentiated liver progenitor cells following down-regulation of YAP1/TAZ and exposure to toxic AhR ligand. Toxicol Lett 280: S132-S133, 2017.

9. Ferraiuolo M, Verduci L, Blandino G and Strano S: Mutant p53 Protein and the Hippo Transducers YAP and TAZ: A critical oncogenic node in human cancers. Int J Mol Sci 18: 961, 2017.

10. Hao Y, Chun A, Cheung K, Rashidi B and Yang X: Tumor suppressor LATS1 is a negative regulator of oncogene YAP. J Biol Chem 283: 5496-5509, 2008.

11. Harvey K and Tapon N: The Salvador-Warts-Hippo pathway-an emerging tumour-suppressor network. Nat Rev Cancer 7: 182-191, 2007.

12. Pan D: The hippo signaling pathway in development and cancer. Dev Cell 19: 491-505, 2010.

13. Varelas X: The Hippo pathway effectors TAZ and YAP in development, homeostasis and disease. Development 141: 1614-1626, 2014.

14. Wang Z, Wu Y, Wang H, Zhang Y, Mei L, Fang X, Zhang X, Zhang F, Chen H, Liu Y, et al: Interplay of mevalonate and Hippo pathways regulates RHAMM transcription via YAP to modulate breast cancer cell motility. Proc Natl Acad Sci USA 111: E89-E98, 2014. 
15. Kilili GK and Kyriakis JM: Mammalian Ste20-like kinase (Mst2) indirectly supports Raf-1/ERK pathway activity via maintenance of protein phosphatase-2A catalytic subunit levels and consequent suppression of inhibitory Raf-1 phosphorylation. J Biol Chem 285: 15076-15087, 2010.

16. Wang C, Thor AD, Moore DH III, Zhao Y, Kerschmann R, Stern R, Watson PH and Turley EA: The overexpression of RHAMM, a hyaluronan-binding protein that regulates ras signaling, correlates with overexpression of mitogen-activated protein kinase and is a significant parameter in breast cancer progression. Clin Cancer Res 4: 567-576, 1998.

17. Zhang J, Ji JY, Yu M, Overholtzer M, Smolen GA, Wang R, Brugge JS, Dyson NJ and Haber DA: YAP-dependent induction of amphiregulin identifies a non-cell-autonomous component of the Hippo pathway. Nat Cell Biol 11: 1444-1450, 2009.

18. Boudreau DM, Yu O and Johnson J: Statin use and cancer risk: A comprehensive review. Expert Opin Drug Saf 9: 603-621, 2010.

19. Gopalan A, Yu W, Sanders BG and Kline K: Simvastatin inhibition of mevalonate pathway induces apoptosis in human breast cancer cells via activation of JNK/CHOP/DR 5 signaling pathway. Cancer Lett 329: 9-16, 2013.

20. Qi XF, Zheng L, Lee KJ, Kim DH, Kim CS, Cai DQ, Wu Z, Qin JW, Yu YH and Kim SK: HMG-CoA reductase inhibitors induce apoptosis of lymphoma cells by promoting ROS generation and regulating Akt, Erk and p38 signals via suppression of mevalonate pathway. Cell Death Dis 4: e518, 2013.

21. Pelaia G, Gallelli L, Renda T, Fratto D, Falcone D, Caraglia M, Busceti MT, Terracciano R, Vatrella A, Maselli R and Savino R: Effects of statins and farnesyl transferase inhibitors on ERK phosphorylation, apoptosis and cell viability in non-small lung cancer cells. Cell Prolif 45: 557-565, 2012.

22. Clendenen TV, Koenig KL, Arslan AA, Lukanova A, Berrino F, Gu Y, Hallmans G, Idahl A, Krogh V, Lokshin AE, et al: Factors associated with inflammation markers, a cross-sectional analysis. Cytokine 56: 769-778, 2011.

23. Fang Z, Tang Y, Fang J, Zhou Z, Xing Z, Guo Z, Guo X, Wang W, Jiao W, Xu Z and Liu Z: Simvastatin inhibits renal cancer cell growth and metastasis via AKT/mTOR, ERK and JAK2/STAT3 pathway. PLoS One 8: e62823, 2013

24. Kochuparambil ST, Al-Husein B, Goc A, Soliman S and Somanath PR: Anticancer efficacy of simvastatin on prostate cancer cells and tumor xenografts is associated with inhibition of Akt and reduced prostate-specific antigen expression. J Pharmacol Exp Ther 336: 496-505, 2011.

25. Ashush H, Rozenszajn LA, Blass M, Barda-Saad M, Azimov D Radnay J, Zipori D and Rosenschein U: Apoptosis induction of human myeloid leukemic cells by ultrasound exposure. Cancer Res 60: 1014-1020,2000.

26. Rosenthal I, Sostaric JZ and Riesz P: Sonodynamic therapy-a review of the synergistic effects of drugs and ultrasound. Ultrason Sonochem 11: 349-363, 2004.

27. Korosoglou G, Hardt SE, Bekeredjian R, Jenne J, Konstantin M, Hagenmueller M, Katus HA and Kuecherer H: Ultrasound exposure can increase the membrane permeability of human neutrophil granulocytes containing microbubbles without causing complete cell destruction. Ultrasound Med Biol 32: 297-303, 2006.

28. Korosoglou G, Behrens S, Bekeredjian R, Hardt S, Hagenmueller M, Dinjus E, Bohm KJ, Unger E, Katus HA and Kuecherer H: The potential of a new stable ultrasound contrast agent for site-specific targeting. An in vitro experiment. Ultrasound Med Biol 32: 1473-1478, 2006.

29. Nie F, Xu HX, Lu MD, Wang Y and Tang Q: Anti-angiogenic gene therapy for hepatocellular carcinoma mediated by microbubble-enhanced ultrasound exposure: An in vivo experimental study. J Drug Target 16: 389-395, 2008

30. Wang Y, Hu B, Diao X and Zhang J: Antitumor effect of microbubbles enhanced by low frequency ultrasound cavitation on prostate carcinoma xenografts in nude mice. Exp Ther Med 3: 187-191, 2012.

31. Xing W, Gang WZ, Yong Z, Yi ZY, Shan XC and Tao RH: Treatment of xenografted ovarian carcinoma using paclitaxel-loaded ultrasound microbubbles. Acad Radiol 15: 1574-1579, 2008.

32. Liu HL, Fan CH, Ting CY and Yeh CK: Combining microbubbles and ultrasound for drug delivery to brain tumors: current progress and overview. Theranostics 4: 432-444, 2014.

33. Unger E, Porter T, Lindner J and Grayburn P: Cardiovascular drug delivery with ultrasound and microbubbles. Adv Drug Deliv Rev 72: 110-126, 2014.
34. Junhua A, Yun J, Zhenzhou W, Ling Y and Ding L: Treatment of malignant liver tumors by radiofrequency ablation combined with low-frequency ultrasound radiation with microbubbles. PLoS One 8: e53351, 2013.

35. Xu WP, Shen E, Bai WK, Wang Y and Hu B: Enhanced antitumor effects of low-frequency ultrasound and microbubbles in combination with simvastatin by downregulating caveolin-1 in prostatic DU145 cells. Oncol Lett 7: 2142-2148, 2014.

36. Yoon YI, Yoon TJ and Lee HJ: Optimization of ultrasound parameters for microbubble-nanoliposome complex-mediated delivery. Ultrasonography 34: 297-303, 2015.

37. Lweesy K, Fraiwan L, Al-Bataineh O, Hamdi N and Dickhaus H: Optimization of ultrasound array designs for high intensity focused treatment of prostate cancer and benign prostatic hyperplasia. Med Biol Eng Comput 47: 635-640, 2009.

38. Feng Y, Tian Z and Wan M: Bioeffects of low-intensity ultrasound in vitro: Apoptosis, protein profile alteration and potential molecular mechanism. J Ultrasound Med 29: 963-974, 2010.

39. Zhang Z, Chen J, Chen L, Yang X, Zhong H, Qi X, Bi Y and $\mathrm{Xu}$ K: Low frequency and intensity ultrasound induces apoptosis of brain glioma in rats mediated by caspase-3, Bcl-2 and survivin. Brain Res 1473: 25-34, 2012.

40. Alberts AW: Lovastatin and simvastatin-inhibitors of HMG CoA reductase and cholesterol biosynthesis. Cardiology 77 (Suppl 4): S14-S21, 1990.

41. Kamigaki M, Sasaki T, Serikawa M, Inoue M, Kobayashi K, Itsuki H, Minami T, Yukutake M, Okazaki A, Ishigaki T, et al: Statins induce apoptosis and inhibit proliferation in cholangiocarcinoma cells. Int J Oncol 39: 561-568, 2011.

42. Toepfer N, Childress C, Parikh A, Rukstalis D and Yang W: Atorvastatin induces autophagy in prostate cancer PC3 cells through activation of LC3 transcription. Cancer Biol Ther 12: 691-699, 2011.

43. Seeger H, Wallwiener D and Mueck AO: Statins can inhibit proliferation of human breast cancer cells in vitro. Exp Clin Endocrinol Diabetes 111: 47-48, 2003.

44. Relja B, Meder F, Wilhelm K, Henrich D, Marzi I and Lehnert M: Simvastatin inhibits cell growth and induces apoptosis and G0/G1 cell cycle arrest in hepatic cancer cells. Int J Mol Med 26: 735-741, 2010.

45. Cho SJ, Kim JS, Kim JM, Lee JY, Jung HC and Song IS: Simvastatin induces apoptosis in human colon cancer cells and in tumor xenografts and attenuates colitis-associated colon cancer in mice. Int J Cancer 123: 951-957, 2008.

46. Koyuturk M, Ersoz M and Altiok N: Simvastatin induces apoptosis in human breast cancer cells: p53 and estrogen receptor independent pathway requiring signalling through JNK. Cancer Lett 250: 220-228, 2007.

47. Chen K and Wang D: Lats1 is the Key Component of Hippo Signaling Pathway: A New Tumor Molecular Biomarker and A Potential Therapeutic Target. Chinese J Cell Biol 37: 256-262, 2015.

48. Jansson L and Larsson J: Normal hematopoietic stem cell function in mice with enforced expression of the Hippo signaling effector YAP1. PLoS One 7: e32013, 2012.

49. Zhi X, Zhao D, Zhou Z, Liu R and Chen C: YAP promotes breast cell proliferation and survival partially through stabilizing the KLF5 transcription factor. Am J Pathol 180: 2452-2461, 2012.

50. Shin SY and Lan Nguyen LK: Unveiling hidden dynamics of hippo signalling: A systems analysis. Genes Bacfl 7: E44, 2016.

51. Castelnovo LF, Bonalume V, Melif S, Ballabio M, Colleoni D and Magnaghi V: Schwann cell development, maturation and regeneration: A focus on classic and emerging intracellular signaling pathways. Neural Regen Res 12: 1013-1023, 2017.

52. Lai D, Ho KC, Hao Y and Yang X: Taxol resistance in breast cancer cells is mediated by the hippo pathway component TAZ and its downstream transcriptional targets Cyr61 and CTGF. Cancer Res 71: 2728-2738, 2011.

53. Mi W, Lin Q, Childress C, Sudol M, Robishaw J, Berlot CH, Shabahang M and Yang W: Geranylgeranylation signals to the Hippo pathway for breast cancer cell proliferation and migration. Oncogene 34: 3095-3106, 2015.

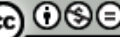

This work is licensed under a Creative Commons Attribution-NonCommercial-NoDerivatives 4.0 International (CC BY-NC-ND 4.0) License. 